\title{
Alimentation azotée et croissance de la fétuque élevée. II. - Absorption de l'azote et distribu- tion dans la plante
}

\author{
François GASTAL $\left({ }^{1}\right)$ \& Bernard SAUGIER \\ Université de Paris-Sud, Laboratoire d'Ecologie végétale, Bâtiment 362, F 91405 Orsay Cedex
}

Ce travail est consacré à l'étude de l'absorption et de la répartition de l'azote chez la fétuque élevée en fonction de sa disponibilité dans le milieu nutritif et de l'intensité de l'éclairement de croissance, tout au long d'une phase de repousse après une coupe.

Les plantes, cultivées en couvert dense, sont soumises à partir de la $2^{\mathrm{e}}$ coupe et selon le traitement, à 2 niveaux de nutrition azotée $(8 \mathrm{mM}$ ou $0,5 \mathrm{mM})$ combinés à 2 niveaux d'éclairement $\left(475 \mu \mathrm{Em}^{-2} \mathrm{~s}^{-1}\right.$ ou $250 \mu \mathrm{Em}^{-2} \mathrm{~s}^{-1}$ ).

En condition de nutrition azotée non limitante, les teneurs en $\mathrm{N}$-Kjeldahl diminuent au cours de la croissance dans les limbes et dans les gaines, de même que les teneurs en $\mathrm{N}$-nitrique et $\mathrm{N}$-organique soluble. Cette baisse, constatée sur l'ensemble du couvert, n'est que partiellement expliquée par la diminution de teneur due au vieillissement des feuilles, la teneur en $\mathrm{N}$-Kjeldahl des limbes émergeant des gaines diminue également pour des ordres d'apparition croissant. Comparée à même biomasse, la teneur en N-Kjeldahl des parties aériennes n'est pas modifiée par l'éclairement aux intensités où nous avons travaillé.

En revanche, les teneurs restent constantes dans les racines pendant toute la repousse.

En condition de disponibilité en azote limitée dans le milieu nutritif, les teneurs en N-nitrique et N-Kjeldah diminuent d'autant plus rapidement que l'éclairement de croissance est plus élevé. Cette baisse concerne aussi bien les limbes émergeant des gaines que les limbes adultes.

La teneur en N-organique soluble est proche de zéro dans les racines du fait de l'arrêt de l'absorption et de l'assimilation. Par contre, c'est dans les limbes qu'elle est le moins affectée par la carence.

Mots clés additionnels : Festuca arundinacea, nitrate, carence azotée, $N$-Kjeldahl, $N$-organique soluble, âge foliaire.

Nitrogen nutrition and growth of tall fescue. II. Nitrogen uptake and partitioning among plant organs.

The absorption and distribution of nitrogen in tall fescue was studied in relation to its availability in the nutrient solution and to light intensity, during a period of growth after cutting. Plants were grown in a dense canopy. After the second cutting they experienced two levels of nitrogen nutrition $(8$ or $0.5 \mathrm{mM})$ combined with two light levels ( 475 or $250 \mu \mathrm{Em}^{-2} \mathrm{~s}^{-1}$ ). When the absorption of nitrogen was not limited by its supply, Kjeldahl-N contents of lamina and sheaths decreased during growth, as well as nitrate- $\mathrm{N}$ and soluble organic$\mathrm{N}$ concentrations. This decrease occurred in the whole canopy. It was only partly explained by the fall in $\mathrm{N}$ content caused by the senescence of individual leaves, since Kjeldahl-N content of emerging laminae also showed a decrease in leaves of increasing rank. For the same biomass, shoot Kjeldahl-N content was not affected by light level at the light intensities used. In contrast, root $\mathrm{N}$ content stayed constant during the whole period of growth ( 6 weeks). When nitrogen supply was limited, nitrate-N and Kjeldahl-N contents decreased more quickly, and the more so when the light level was higher. This decrease was seen in emerging laminae as well as in mature ones. Soluble organic- $\mathrm{N}$ content was near zero in roots, since $\mathrm{N}$ absorption and assimilation was much decreased. However, lamina $\mathrm{N}$ stayed more constant in conditions of limiting $\mathrm{N}$ supply.

Additional key words : Festuca arundinacea, nitrate, nitrogen stress, Kjeldahl- $N$, soluble organic $N$, leaf age. 


\section{INTRODUCTION}

L'étude des besoins en azote d'une prairie passe par la détermination des flux d'azote entre les différents compartiments de cet écosystème, en particulier, du flux d'azote exporté du système sol-racines vers les parties aériennes des plantes. De nombreux travaux antérieurs ont montré que ce flux dépend de la disponibilité en azote au voisinage des racines. Toutefois ce facteur, s'il a été le plus étudié, n'est pas le seul en jeu. La vitesse de croissance des plantes est au moins aussi déterminante. Ceci est illustré au champ : la relation entre biomasse aérienne produite et quantité d'azote " exporté » est indépendante de la durée de réalisation de cette biomasse pourvu que la fertilisation soit portée à un niveau «non limitant de la production » (LEMAIRE \& SALETTE, 1984). Cette relation montre qu'au champ, les prélèvements en azote par les parties aériennes ne sont pas constants, mais diminuent au cours de la croissance (DARWINKEL, 1975; NyE \& Tinker, 1977 ; SAletTE \& Lemaire, 1981 ; PENNING de VRIES, 1982). Son intérêt agronomique nécessite que les bases physiologiques qui lui sont sous-jacentes soient clairement définies, ce qui n'est pas le cas en situation de terrain du fait d'une disponibilité en azote du sol difficilement contrôlable et probablement non optimale en fin de croissance.

L'objectif de ce travail, qui s'insère dans une étude plus large des relations entre assimilation de l'azote, assimilation du carbone et croissance de la fétuque élevée, est double. D'une part, il s'agit de séparer sans ambiguïté une situation de nutrition azotée limitante d'une situation non limitante afin de déterminer l'importance relative de chacune dans la diminution de teneur en azote au cours de la croissance, d'autre part, de vérifier l'unicité de la relation entre teneur en azote et biomasse aérienne produite à niveau de nutrition non limitante et d'en préciser l'origine et les limites d'utilisation, par une étude en condition contrôlée.

\section{MATÉRIEL ET MÉTHODES}

\section{A. Conditions de culture}

Les conditions de culture ont été décrites en détail dans la $1^{\text {re }}$ partie de cette étude (GASTAL \& SAUGiER, 1986). Rappelons en brièvement les points principaux.

L'ensemble des expériences comporte 3 essais successifs. Pour chacun d'eux, les plantes sont cultivées à $22{ }^{\circ} \mathrm{C}$, sur milieu hydroponique, de la germination à la $1^{\text {re }}$ coupe, puis durant la $1^{\text {re }}$ repousse jusqu'à la $2^{\mathrm{e}}$ coupe, à même éclairement $(E)$ et même niveau de nutrition azotée $(\mathrm{N})$ (voir tabl. 1). A ce moment, elles sont séparées en 3 lots de 42 plantes chacun, et la $2^{\mathrm{e}}$ repousse s'effectue, selon le traitement, à éclairement $\mathrm{E}$ ou e et nutrition azotée $\mathrm{N}$ ou $\mathrm{n}$ (respectivement niveaux élevés $(E, N)$ ou niveaux faibles $(e, n)$ d'éclairement ou de nutrition azotée, $\mathrm{N}=8 \mathrm{mM}$ et $\mathrm{n}=0,5 \mathrm{mM}($ tabl. 1)). Les conditions de croissance imposées pour chaque traitement de chacun des 3 essais sont indiquées dans le tableau 2 . $\mathrm{N}-\mathrm{n}$ signifie 15 jours à $N$ puis passage à $n$. Une photopériode de $14 \mathrm{~h}$ permet d'éviter la mise à fleur. Tous les 7 à $11 \mathrm{j}, 5$ plantes de chaque traitement sont prélevées, séparées en limbes, gaines, chaumes et racines,
TABI.EAU 1

Intensité des éclairements de croissance $\left(\mu \mathrm{Em}^{-2}{ }_{s}{ }^{\prime}\right.$ et composition des solutions nutritives pendant les cultures. Light levels during growth $\left(\mu \mathrm{Em}^{-2} \mathrm{~s}^{-1}\right)$ and composition of mutrient solutions.

Eclairement de croissance :

\begin{tabular}{lcc} 
& $\begin{array}{c}30 \mathrm{~cm} \text { au-dessus } \\
\text { du collet }\end{array}$ & $\begin{array}{c}\text { hauteur } \\
\text { du collet }\end{array}$ \\
\hline Eclairement $\mathrm{E}$ & 450 & 310 \\
\hline Eclairement $\mathrm{e}$ & 250 & 180 \\
\hline
\end{tabular}

Solutions mutritives (meq/l) :

\begin{tabular}{lcc} 
& & \\
& $\mathrm{N}: 8$ meq N/1 & $\mathrm{n}: 0,5 \mathrm{meq} / 1$ \\
\hline $\mathrm{KNO}_{3}$ & 1,9 & 0,05 \\
$\left.\mathrm{Ca}_{3} \mathrm{NO}_{3}\right)_{2}$ & 1,1 & 0,15 \\
$\mathrm{NO}_{3} \mathrm{NH}_{4}$ & 2,5 & 0,15 \\
$\mathrm{CaCl}_{2}$ & 0,5 & 1,45 \\
$\mathrm{NaCl}$ & 0,1 & - \\
$\mathrm{MgSO}_{4}$ & 0,5 & 0,5 \\
$\mathrm{~K}_{2} \mathrm{SO}_{4}$ & - & 0,7 \\
$\mathrm{Na}_{2} \mathrm{SO}_{4}$ & - & 0,1 \\
$\mathrm{PO}_{4} \mathrm{H}_{2} \mathrm{~K}$ & 0,4 & 1,05 \\
$\mathrm{PO}_{4} \mathrm{HK}_{2}$ & 0,3 & 0,8 \\
\hline \hline
\end{tabular}

TABLEAU 2

Conditions d'éclairement et de nutrition azotée dans les différents traitements.

Light levels and nitrogen concentrations in the various treatments.

$1^{\mathrm{cr}}$ traitement $2^{\mathrm{c}}$ traitement $3^{\mathrm{c}}$ traitement

\begin{tabular}{llll}
\hline Essai 1 & $(E, N)$ & $(E, N-n)$ & $(E, n)$ \\
\hline Essai 2 & $(E, N)$ & $(e, N)$ & $(e, n)$ \\
\hline Essai 3 & $(E, N)$ & $(E, n)$ & $(e, N)$ \\
\hline
\end{tabular}

puis séchées à $65^{\circ} \mathrm{C}$. Lors de l'essai 3 , les limbes sont séparés en 4 classes d'âge croissant de $\mathrm{L}_{1}$ à $\mathrm{L}_{4}$ :

$\mathrm{L}_{1}$ : limbes émergeant des gaines, dont la longueur est inférieure à $10 \mathrm{~cm}$;

$\mathrm{L}_{2}$ : limbes en cours de développement, dont la longueur est supérieure à $10 \mathrm{~cm}$;

$\mathrm{L}_{3}$ : limbes développés, à extrémité non sectionnée par la dernière coupe ;

$\mathrm{L}_{4}$ : limbes développés, à extrémité sectionnée (limbes les plus âgés puisqu'ils étaient contenus dans les gaines lors de la dernière coupe).

\section{B. Analyses d'azote sur les poudres végétales}

\section{N-Kjeldahl}

Après minéralisation par le procédé Kjeldahl, les teneurs sont déterminées par dosage colorimétrique de l'ammonium (méthode au salicylate de sodium en milieu alcalin en présence de chlore) sur système automatique Technicon. 


\section{N-soluble}

L'azote soluble est extrait de la poudre végétale dans un tampon $\left(\mathrm{KH}_{2} \mathrm{PO}_{4}, \mathrm{Na}_{2} \mathrm{HPO}_{4}, 12 \mathrm{H}_{2} \mathrm{O}, \mathrm{pH} 7\right)$ pendant $1 \mathrm{~h}$. Après filtration, le nitrate est dosé par la méthode de GRIESS (réduction du nitrate en nitrite par le sulfate d'hydrazine à $37^{\circ} \mathrm{C}$ en présence de sulfate de cuivre et dosage colorimétrique du complexe nitrite, sulfanilamide et (n-naphtyl) éthylène diammonium dichlorure à $520 \mathrm{~nm}$ ). Par ailleurs, le filtrat obtenu est repris pour le dosage de la fraction organique soluble de l'azote par le procédé Kjeldahl. Cette méthode de dosage de $\mathrm{N}$-organique soluble présente l'inconvénient d'une incertitude dans le cas de poudre végétale à teneur élevée en nitrate du fait d'interférences possibles (surestimation de $\mathrm{N}$-organique soluble).

D'autre part, la solution d'extraction choisie n'est pas exactement équivalente à celle adoptée classiquement en physiologie végétale (mélange hydroalcoolique) mais son utilisation permet de mieux approcher la qualité azotée des graminées pour les ruminants.

\section{RÉSULTATS}

\section{A. Assimilation de l'azote en conditions de nutrition non limitante $(\mathbf{N})$}

Dans le traitement à forte teneur en azote de la solution nutritive et à éclairement élevé (E, N) les teneurs en $\mathrm{N}$-Kjeldahl diminuent régulièrement dans les limbes et dans les gaines, passant de 5 à 3,7 p. 100 pour les premiers, et de 3,6 à 2,3 p. 100 pour les secondes de la $1^{\text {re }}$ à la $6^{\mathrm{e}}$ semaine de repousse (fig. 1).

Dans les chaumes et les racines, ces teneurs sont plus basses, mais restent constantes pendant toute la période de croissance. Ceci confirme qu'aucune limitation minérale accidentelle n'est survenue durant les expériences.

La diminution de teneur observée dans les limbes et dans les gaines est donc bien un phénomène propre à ces 2 compartiments de la plante entière. Il est important de préciser que toutes nos analyses chimiques sur les limbes ont été effectuées sur les parties vertes, après séparation des limbes sénescents et des parties « jaunes ». Des résultats similaires ont été observés en conditions contrôlées par GOUNOT (1982), CALOIN \& YU (1984) sur dactyle et par ROBSON \& DEACON (1978) sur Lolium perenne.

Afin de déterminer si cette diminution de teneur en $\mathrm{N}$-Kjeldahl concerne une forme particulière ou l'ensemble des composés azotés, nous avons été conduits à séparer azote nitrique et azote organique soluble de la matière sèche. Ceci permet de constater (fig. 2) que ces 2 fractions, exprimées en pourcentage de la matière sèche, diminuent elles aussi dans les limbes mais dans un rapport qui semble un peu plus faible (15 à $20 \mathrm{p} .100)$ que pour N-Kjeldahl (25 à 30 p. 100) en 6 semaines de repousse. La baisse de teneur en $\mathrm{N}$-nitrique et $\mathrm{N}$-organique soluble ne suffit pas à expliquer la diminution de N-Kjeldahl, dont la composante la plus importante doit donc être attribuée à une baisse de teneur de la fraction $\mathrm{N}$-organique insoluble.

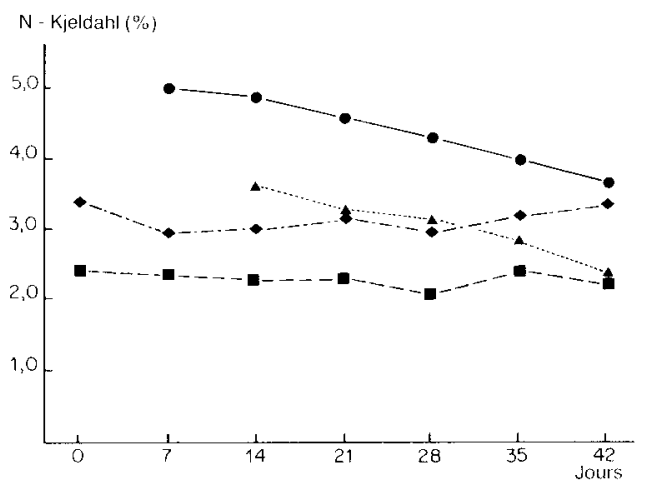

Figure 1

Evolution des teneurs en $N$-Kjeldahl dans les limbes (•), gaines $(\mathbf{\Delta})$, chaumes $(\bullet)$ et racines ( $\mathbf{\square})$ au cours de la croissance à éclairement $E$ et nutrition azotée non limitante $N$.

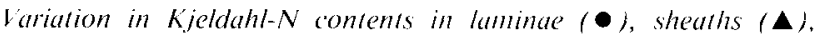

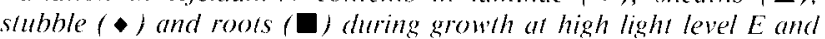
high nitrogen concentration $N$.

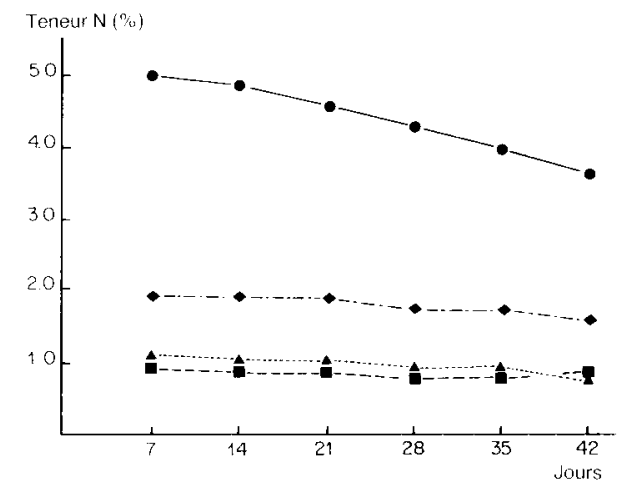

Figure 2

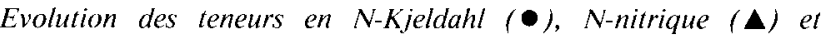
$N$-organique soluble (ם) ( $: N$-nitrique $+N$-organique soluble) dans les limbes au cours de la croissance à éclairement $E$ et nutrition azotée non limitante $N$.

Variation in several $N$ contents of the lamina at light level $E$, and high nitrogen concentration $N$.

- : Kjeldahl-N ; $\boldsymbol{\Delta}:$ Nitrate- $N$;

- : Nitrate-N + Soluble organic-N.

: Soluble organic- $N$;

$$
\text { - Nirrare-N t Soluble organic-N. }
$$

On a souvent signalé une baisse de la teneur en azote d'une feuille au cours de son vieillissement (Robson \& DEACON, 1978 ; PATE \& AtKInS, 1983). Nous avons donc cherché à déterminer si ce phénomène, combiné à l'augmentation de l'âge moyen des feuilles du couvert au cours de la repousse, pouvait expliquer la diminution globale de la teneur en $\mathrm{N}$ Kjeldahl observée.

Dans ce but, nous avons séparé, dans l'essai 3, les limbes verts, à chaque prélèvement, en 4 classes d'âge $\mathrm{L}_{1}, \mathrm{~L}_{2}, \mathrm{~L}_{3}$ et $\mathrm{L}_{4}$ (âge croissant) de la manière indiquée au paragraphe précédent. Les analyses de teneur en $\mathrm{N}$ Kjeldahl sur ces échantillons (fig. 3) montrent en général, pour une date de prélèvement donnée, une baisse de la teneur avec l'augmentation de l'âge. Toutefois, l'écart de teneur entre les classes $\mathrm{L}_{1}$ et $\mathrm{L}_{4}$ à une date est, quel que soit le prélèvement, plus petit que la diminution de teneur globale de l'ensemble des limbes du couvert observée dans la figure 1. La teneur en $\mathrm{N}$ Kjeldahl diminue également dans la classe $\mathrm{L}_{1}$ au cours 


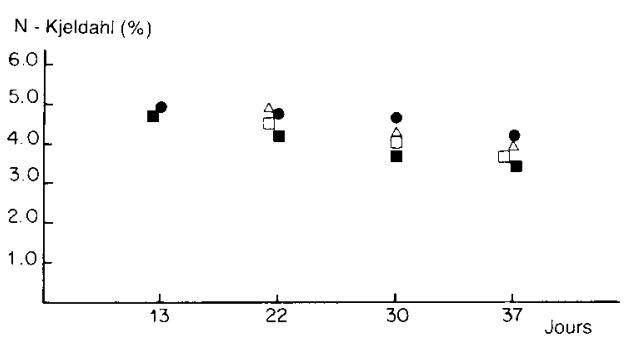

Figure 3

Evolution au cours de la croissance de la teneur en N-Kjeldahl des limbes séparés en quatre classes d'âge (âge croissant de $L_{1} \grave{a} L_{4}$ ) à éclairement $E$ et nutrition azotée non limitante $N$.

- : $L_{1}-\triangle: L_{2}-\square: L_{3}-\mathbf{\square}: L_{4}$.

Variation during growth of Kjeldahl-N contents of several age classes of laminae at high light level $E$, and high nitrogen concentration $N$. $L_{1}$ to $L_{4}$ : increasing age $\left(L_{1}:\right.$ leaves emerging from the sheath).

de la croissance. L'effet de l'âge des limbes sur leur teneur ne peut donc expliquer à lui seul la baisse enregistrée sur le couvert au cours de la croissance.

\section{B. Assimilation de l'azote en conditions de nutrition limitante (n)}

La figure 4 montre l'évolution des teneurs en $\mathrm{N}$ nitrique, $\mathrm{N}$-organique soluble et $\mathrm{N}$-Kjeldahl des 4 compartiments de plante que nous avons distingués.

Le transfert sur milieu carencé entraîne dans chaque cas une diminution d'allure exponentielle de la teneur en $\mathrm{N}$-Kjeldahl ; parallèlement, la teneur en $\mathrm{N}$-nitrique baisse très rapidement et devient négligeable après 10 à $20 \mathrm{j}$ suivant l'organe considéré et la vitesse de croissance de la plante entière, excepté dans les chaumes où elle se maintient à 0 ou 0,05 p. 100 (il peut s'agir du nitrate accumulé dans les chaumes morts lors des coupes précédentes et qui n'est pas redistribué). Ceci correspond au schéma classique assignant au nitrate un rôle de molécule azotée de réserve, localisée pour partie dans le cytoplasme et pour partie dans les vacuoles, ce dernier pool étant prépondérant dans ces conditions de forte teneur (ROBIN et al., 1983).

Il est intéressant, dans la mesure où l'attention a plus rarement été portée sur ce point, de comparer l'évolution des teneurs en $\mathrm{N}$-organique soluble entre les différentes parties de la plante lors de la mise en carence. Une remarquable stabilité de cette fraction azotée est à noter, d'une part, dans les limbes vers 0,6 à 0,5 p. 100 de la $3^{\mathrm{e}}$ à la $6^{\mathrm{e}}$ semaine pour $(\mathrm{E}, \mathrm{n})$ et de la $5^{\mathrm{e}}$ à la $6^{\mathrm{e}}$ semaine pour $(\mathrm{E}, \mathrm{N}-\mathrm{n})$ (fig. 4-1), d'autre part, dans les gaines, à ces mêmes dates et à une teneur plus basse, 0,4 à 0,5 p. 100 (fig. 4-2). Dans les racines, la diminution plus régulière sur la période de repousse permet d'atteindre une valeur de 0,15 p. 100 après $42 \mathrm{j}$ (fig. 4-4). La comparaison entre traitements témoin et carencé pour cette même fraction azotée indique, en fin de repousse, une différence relativement peu marquée dans les limbes ( - 38 p. 100 par rapport à $(E, N)$ ), intermédiaire dans les gaines et importante dans les chaumes et les racines $(-66$ p. 100 et -79 p. 100 respectivement par rapport à $(\mathrm{E}, \mathrm{N})$ ).

Les teneurs en N-Kjeldahl mesurées sur les limbes des différentes classes d'âge $L_{1}$ à $L_{4}$ (fig. 5) indiquent, comme dans le cas de nutrition azotée $\mathrm{N}$, peu de différence entre les limbes qui émergent des gaines $\left(L_{1}\right)$ et les limbes les plus âgés $\left(\mathrm{L}_{4}\right)$.

\section{Evolution des teneurs dans les limbes morts}

Les tableaux 3 et 4 permettent de comparer les teneurs en azote des limbes sénescents et des limbes vivants prélevés à la même date et sur le même échantillon. Ce mode de comparaison n'apporte qu'une indication qualitative sur l'azote remobilisé à la sénescence dans les différentes conditions de croissance. En effet, les analyses effectuées par rapport à la matière sèche ne prennent en compte ni le carbone qui lui aussi a pu être remobilisé, ni le carbone perdu par respiration lors des processus de sénescence, phénomènes conduisant à une diminution importante à la masse surfacique.

A éclairement fort $(E)$, les teneurs en N-Kjeldahl dans les limbes morts des plantes cultivées sur milieu carencé sont diminuées en valeur absolue par rapport aux plantes bien alimentées (tabl. 3). Toutefois, comparées en valeur relative à la teneur des limbes verts du même échantillon, les différences entre traitement $\mathrm{N}, \mathrm{n}$ ou $\mathrm{N}-\mathrm{n}$ disparaissent. En revanche, à éclairement plus réduit (e) (tabl. 4), les teneurs en $\mathrm{N}$-Kjeldahl des limbes à la sénescence sont légèrement supérieures à celles des limbes verts.

En ce qui concerne la fraction $\mathrm{N}$-nitrique, les teneurs des limbes morts sont systématiquement plus élevées que celles des limbes encore vivants (tabl. 3), ce qui peut s'expliquer par l'arrêt du métabolisme azoté à la sénescence.

TABLEAU 3

Teneur en $N$-Kjeldahl et $N$-nitrique dans les limbes sénescents (essai 1$)$.

Kjeldahl-N and nitrate- $N$ contents in senescing laminae (trial 1).

$35 \mathrm{j}$ après coupe

\begin{tabular}{|c|c|c|c|c|c|c|c|c|}
\hline & \multicolumn{2}{|c|}{ N-Kjeldhal } & \multicolumn{2}{|c|}{$\mathrm{N}$-nitrique } & \multicolumn{2}{|c|}{ N-Kjeldhal } & \multicolumn{2}{|c|}{$\mathrm{N}$-nitrique } \\
\hline & $\begin{array}{l}\text { Limbes } \\
\text { sénescents }\end{array}$ & $\begin{array}{l}\text { Limbes } \\
\text { verts }\end{array}$ & $\begin{array}{l}\text { Limbes } \\
\text { sénescents }\end{array}$ & $\begin{array}{l}\text { Limbes } \\
\text { verts }\end{array}$ & $\begin{array}{l}\text { Limbes } \\
\text { sénescents }\end{array}$ & $\begin{array}{l}\text { Limbes } \\
\text { verts }\end{array}$ & $\begin{array}{l}\text { Limbes } \\
\text { sénescents }\end{array}$ & $\begin{array}{c}\text { Limbes } \\
\text { verts }\end{array}$ \\
\hline$(E, N)$ & 2,79 & 3,98 & 1,28 & 1,00 & 2,55 & 3,70 & 1,46 & 0,77 \\
\hline$(E, n)$ & - & - & 0 & 0,04 & 1,24 & 1,83 & 0 & 0,02 \\
\hline$(\mathrm{E}, \mathrm{N}-\mathrm{n})$ & 1,46 & 2,50 & 0,17 & 0,04 & 1,01 & 2,04 & 0,25 & 0,05 \\
\hline
\end{tabular}



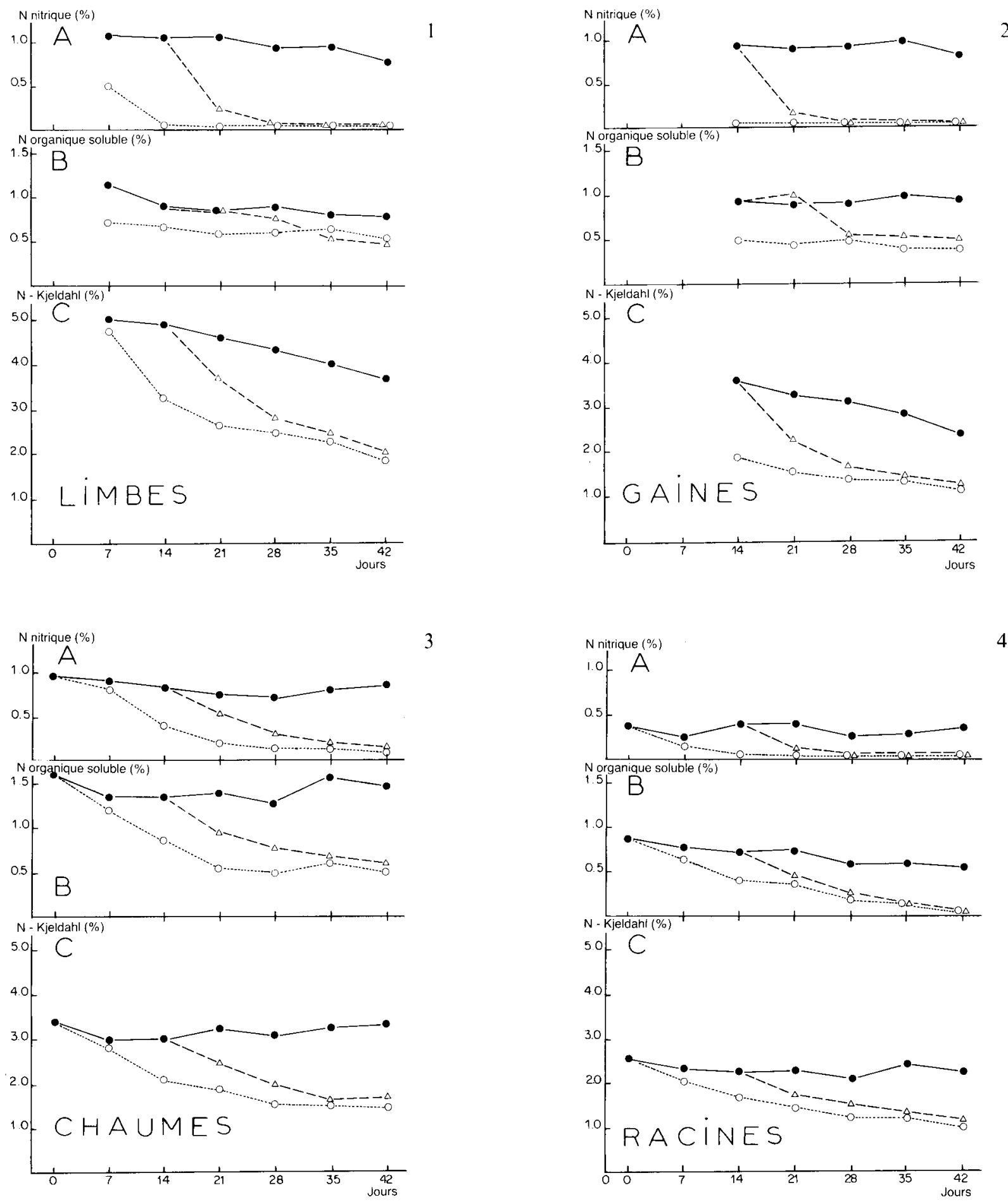

Figure 4

Evolution des teneurs $N$-nitrique (A), $N$-organique soluble (B) et $N$ Kjeldahl (C) au cours de la croissance selon le niveau de nutrition azotée à éclairement $E$.

gen nutrition $(N=$ high, $n=$ low) at high light level.
$A$ - Nitrate- $N$
$B$ - Soluble organic- $N \quad \triangle:(E, N-n)$
$C-$ Kjeldahl-N $O:(E, n)$.

\section{DISCUSSION}

Le choix de la concentration en azote du milieu nutritif $\mathrm{N}(8 \mathrm{mM})$ a permis, avec la fréquence de renouvellement adoptée, d'obtenir une variation de cette valeur entre 2 renouvellements inférieure à 50 p. 100 par rapport à la concentration initiale, par suite de l'effet conjugué de l'absorption racinaire et de l'évapotranspiration. Ces conditions expérimenta-

les permettent de maintenir un niveau de nutrition azotée non limitante, puisqu'on a montré, sur graminée, que l'absorption de l'azote est indépendante de sa concentration dans la solution nutritive au-dessus d'une valeur d'environ 0,05 mM (CLEMENT et al., 1978 ; TRIBOÏ-BLONDEL, 1979).

En revanche, dans le cas du traitement $\mathrm{n}(0,5 \mathrm{mM})$, la concentration en azote du milieu nutritif diminue rapidement et peut s'annuler avant le renouvellement. 


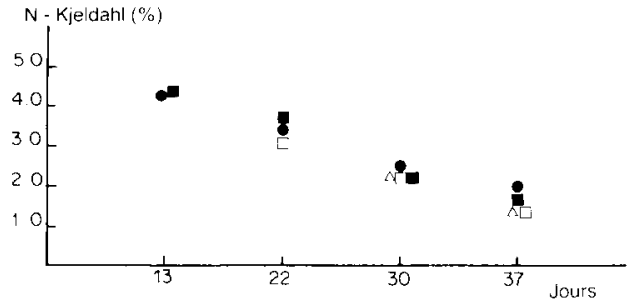

Figure 5

Evolution au cours de la croissance des teneurs en $N$-Kjeldahl des limbes séparés en quatre classes d'âge (âge croissant de $L_{1}$ à $L_{4}$ ) à éclairement $E$ et nutrition azotée limitante $n$.

- : $L_{1}-\wedge: L_{2}-\square: L_{3}-\mathbf{\square}: L_{4}$.

Variation during growth of Kjeldahl- $N$ contents of several age classes of lamina (increasing age from $L_{1}$ to $L_{4}$ ) at high light level $E$ and low nitrogen concentration $n$.

TABLEAU 4

Teneur en N-Kjeldahl dans les limbes sénescents (essai 3). Kjeldahl-N content in senescing laminae (trial 3 ).

\begin{tabular}{lcccc} 
& \multicolumn{2}{c}{} & \multicolumn{2}{c}{$\mathrm{L}_{4}$} \\
\cline { 2 - 5 } & $\begin{array}{c}\text { Limbes } \\
\text { sénescents }\end{array}$ & $\begin{array}{c}\text { Limbes } \\
\text { verts }\end{array}$ & $\begin{array}{c}\text { Limbes } \\
\text { sénescents }\end{array}$ & $\begin{array}{c}\text { Limbes } \\
\text { verts }\end{array}$ \\
\hline$(\mathrm{E}, \mathrm{N})$ & 3,21 & 3,78 & 3,07 & 3,79 \\
\hline$(\mathrm{E}, \mathrm{n})$ & 1,04 & 1,28 & 1,28 & 1,68 \\
\hline$(\mathrm{e}, \mathrm{N})$ & 3,81 & 3,66 & 3,81 & 3,66 \\
\hline \hline
\end{tabular}

Dans ce dernier cas, l'azote absorbé est la totalité de l'azote disponible dans le milieu de culture. Ce protocole expérimental permet, en modulant le volume de solution disponible ou sa concentration, de définir la sévérité de la carence que l'on désire imposer.

Dans nos essais, les différences d'absorption entre les traitements peuvent être évaluées à partir des analyses de $\mathrm{N}$-Kjeldahl sur le matériel végétal et des mesures de biomasse. En comparaison à ces valeurs (fig. 6) sont indiquées les quantités d'azote disponible par plante dans le milieu pour chaque traitement (valeurs cumulées depuis la coupe), calculées comme la somme des quantités apportées à chaque renouvellement divisée par le nombre de plantes restant dans chaque bac, compte tenu des prélèvements antérieurs. Ainsi, au $42^{\mathrm{e}} \mathrm{j}$ de repousse, l'absorption d'azote pour $(E, n)$ a été de 30 p. 100 de celle du témoin $(E, N)$, et celle de $(E, N-n)$ de 42 p. 100 de ce même traitement de référence $(\mathrm{E}, \mathrm{N})$.

Le protocole adopté n'est toutefois pas parfait puisque le rapport de l'azote absorbé par les plantes du milieu $\mathrm{N}$ à l'azote absorbé par celles du milieu n n'est pas contrôlé et varie au cours de la croissance. Un ajustement journalier des apports d'azote pour les traitements carencés à une fraction de la consommation des plantes bien alimentées serait une amélioration technique intéressante (INGESTAD \& LUND, 1979).

La mise en carence entraîne la disparition presque totale du nitrate dans les limbes en 10 à $15 \mathrm{j}$. Cependant au champ, les teneurs en $\mathrm{N}$-nitrique des graminées fourragères sont très faibles, sauf dans la semaine qui suit une fertilisation azotée si elle est

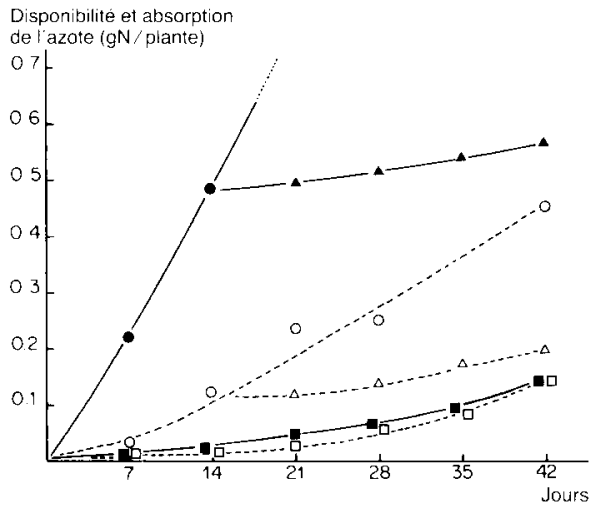

Figure 6

Comparaison des quantités d'azote disponibles dans le milieu nutritif et des quantités absorbées par les plantes durant leur croissance (Essai l) (quantités cumulées en $g \mathrm{~N} /$ plante)

azote disponible : $:(E, N)-\quad \mathbf{\Delta}:(E, N-n)-\quad \mathbf{v}:(E, n)$ azote absorbé $: \bigcirc:(E, N)-\quad \triangle:(E, N-n)-\quad \square:(E, n)$

Comparison of nitrogen availability in the growth medium and nitrogen uptake by plants (cumulated amounts, $g \mathrm{~N} /$ plant).

importante (SALETTE, comm. pers.). La comparaison de la physiologie des plantes du laboratoire avec celles du terrain nécessite donc une certaine prudence du fait du rôle de réserve et $\mathrm{du}$ rôle osmotique possible du nitrate accumulé dans les premières.

La fraction $\mathrm{N}$-organique soluble a parfois été considérée comme un pool d'azote de réserve. Les résultats présentés indiquent qu'il n'en est rien, au moins en ce qui concerne les limbes, et que l'évolution de cette fraction de l'azote lors d'une carence en cet élément dépend de l'organe considéré. Dans les racines, elle diminue de manière très marquée jusqu'à une valeur faible (inférieure à 0,1 p. 100), ce qui s'explique par le ralentissement important de l'absorption et de l'assimilation. Ce pool est maintenu à un niveau plus important dans les chaumes et les gaines, qui sont les voies de passage obligatoire de l'azote lors des remobilisations entre feuilles sénescentes et feuilles plus jeunes. Dans les limbes, la diminution de teneur de cette fraction $\mathrm{N}$-organique soluble est la plus limitée. Cette observation peut être rapprochée d'autres faites sur les mêmes plantes, qui indiquaient que, si la croissance des limbes est ralentie par la carence en azote (d'après l'évolution de l'indice foliaire), l'assimilation du carbone, elle, n'est pas sensiblement modifiée en comparaison avec celle des plantes bien alimentées. Les activités de protéosynthèse, protéolyse et métabolisme de l'azote associés au métabolisme du carbone, maintenu lors de la carence en azote, offrent une explication possible à la diminution peu marquée de $\mathrm{N}$-organique soluble dans les limbes.

Compte tenu de la diminution de la teneur en $\mathrm{N}$ Kjeldahl au cours de la croissance sans limitation de la disponibilité en azote, il est impératif, pour juger de l'effet d'un traitement particulier, de comparer les teneurs à égalité de biomasse produite et non pas à même temps de croissance (LEMAIRE \& SALETTE, 1984). Cette représentation (fig. 7) permet de rassembler sur une même courbe les points obtenus à partir des traitements témoins $(\mathrm{E}, \mathrm{N})$ et du traitement à éclairement plus réduit mais nutrition azotée non limitante $(e, N)$. La similitude des relations entre la teneur en $\mathrm{N}$-Kjeldahl et la biomasse aérienne obtenues à ces 


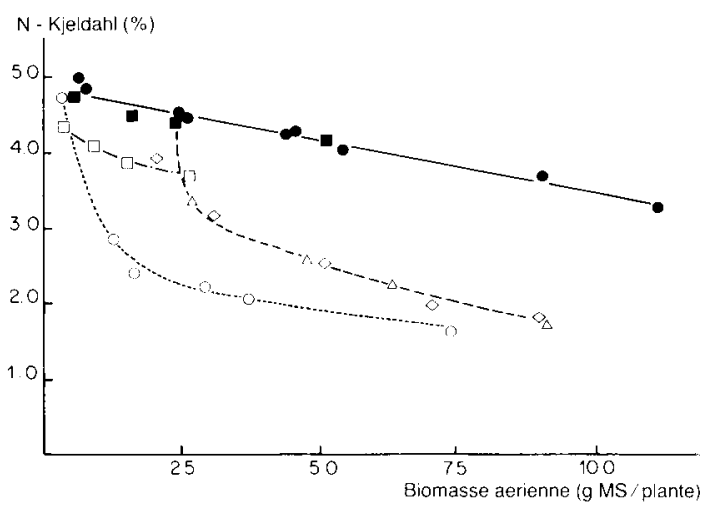

Figure 7

Evolution de la teneur en $\mathrm{N}$-Kjeldahl des parties aériennes en fonction de leur biomasse au cours de la croissance (essai 1 et essai 2$)$. $\bullet:(E, N)-\triangle:(E, N-n)-O:(E, n)-\boldsymbol{\square}:(e, N)-\square:(e, n)-$ $\diamond:$ d'après LEMAIRE \& SALETTE (1984).

Variation in shoot Kjeldahl- $N$ content in relation to biomass per unit ground area during growth.

2 éclairements permet de définir, dans la plage d'intensité lumineuse où nous avons travaillé, une relation unique, indépendante de la vitesse de croissance, à niveau de nutrition azotée non limitante. Tout écart à cette courbe (par défaut) correspond à une diminution de la disponibilité en azote du milieu nutritif. Au niveau physiologique, ceci implique que l'absorption de l'azote soit étroitement ajustée à l'assimilation du carbone.

L'analyse de l'évolution de la teneur en N-Kjeldahl au cours de la croissance, en absence de limitation minérale, conduit à s'interroger sur les causes de sa diminution dans les limbes verts. Il nous semble important de rappeler ici qu'une teneur est une valeur relative, qui dépend également de la teneur des autres éléments dans la matière sèche, en particulier de la teneur en carbone.

Une $1^{\text {re }}$ explication est à rechercher dans l'augmentation de l'âge moyen de l'ensemble des limbes du couvert, puisque les limbes les plus développés $\left(\mathrm{L}_{4}\right)$ ont une teneur inférieure à celle des limbes les plus jeunes $\left(\mathrm{L}_{1}\right)$. Sur ce point, plusieurs hypothèses peuvent être examinées :

- synthèse des constituants pariétaux (cellulose, hémi-cellulose, lignine) et/ou accumulation de composés carbonés de réserve (DEINUM, 1984), depuis la fin de l'élongation jusqu'à la sénescence, qui s'accorderait avec l'augmentation de masse surfacique observée ;

- remobilisation de l'azote plus importante que celle du carbone lors des premières étapes de la sénescence (PATE \& ATKINS, 1983).

Mais il est également nécessaire de prendre en compte un effet lié à l'ordre d'apparition des limbes. Ceci est confirmé indirectement par DEINUM (1984) qui indique une augmentation de la teneur en constituants pariétaux à maturité pour des numéros d'ordre d'apparition des limbes croissants.

Devant l'impossibilité d'expliquer de manière satisfaisante la diminution de teneur en N-Kjeldahl au cours de la croissance, 2 observations méritent d'être soulignées, dans la mesure où elles suggèrent un critère permettant de s'affranchir de la mesure de biomasse nécessaire pour interpréter la teneur en azote.
D'une part, les mesures de masse surfacique rapportant l'azote non plus à la matière sèche mais à la surface foliaire, permettent de s'affranchir des variations de la composition en éléments autres que l'azote. On constate alors que la quantité d'azote par unité de surface foliaire, mesurée globalement sur le couvert, ne varie pas pendant la croissance en azote non limitant (fig. 8A). Ce point est en accord avec l'hypothèse formulée plus haut à partir des travaux de DEINUM (1984) mettant en rapport la diminution de teneur en $\mathrm{N}$-Kjeldahl dans les limbes et une éventuelle augmentation de la teneur en constituants pariétaux et/ou composés carbonés de réserve. D'autre part, on constate au cours de la repousse une diminution de la teneur en eau des limbes et le rapport quantité d'azote/quantité d'eau dans les feuilles reste constant pendant la croissance (fig. 8B). Ce paramètre, pour être mesuré au champ, nécessiterait cependant la détermination de la teneur en eau après réhydratation rapide des prélèvements, de manière à s'affranchir des variations dues à l'état hydrique du sol ou à la demande évaporative.

En comparaison avec nos données de laboratoire, nous avons indiqué dans la figure 7 les valeurs publiées par LEMAIRE \& SALETTE (1984) obtenues au champ avec une fertilisation en azote définie par ces auteurs comme "non limitante ". Pour cela, les données de notre essai ont été rapportées à l'unité de surface de sol, ce qui constitue une approximation compte tenu de la faible surface occupée par nos échantillons. En dépit de cette imprécision, il apparaît que l'évolution de la teneur en $\mathrm{N}$-Kjeldahl dans leur expérimentation se rapproche de celle du traitement $(\mathrm{E}, \mathrm{N}-\mathrm{n})$. Ainsi, même avec un apport minéral important en début de croissance, la disponibilité en azote du sol n'a pu se maintenir à un niveau non limi-

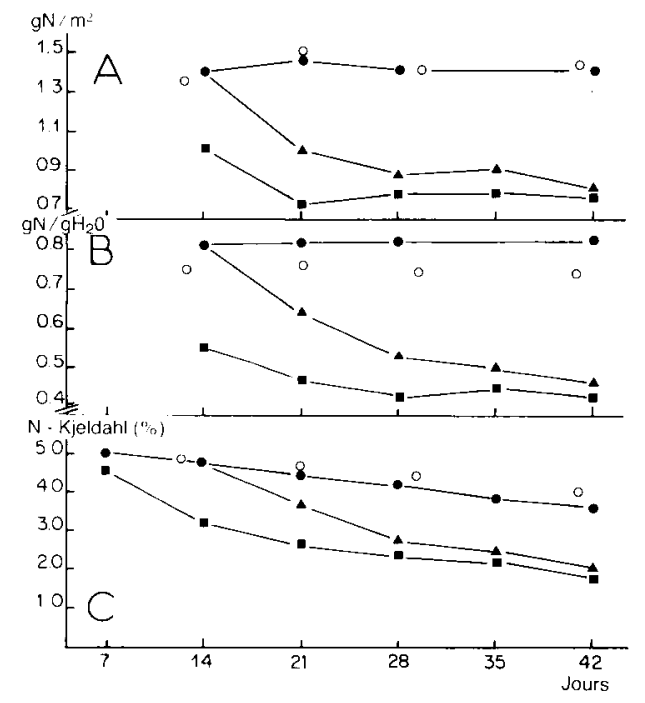

Figure 8

$8 A$ : Evolution du rapport quantité $N$-Kjeldahl/surface foliaire dans les limbes $\left(\mathrm{g} \mathrm{N} / \mathrm{m}^{2}\right)$ à éclairement $E$.

$8 A$ : Kjeldahl- $N$ content/leaf area $\left(g \mathrm{~N} / \mathrm{m}^{2}\right)$ in lamina.

$8 B$ : Evolution du rapport quantité $N$-Kjeldahl/quantité d'eau dans les limbes $\left(\mathrm{mg} \mathrm{N} / \mathrm{mg} \mathrm{H}_{2} \mathrm{O}\right)$ à éclairement $E$.

$8 B$ : Kjeldahl-N content/water content ( $m g \mathrm{~N} / \mathrm{mg} \mathrm{H}_{2} \mathrm{O}$ ) in lamina. $8 C$ : Rappel des teneurs en $N$-Kjeldahl dans les traitements correspondants.

- :(E,N) essai $1-O:(E, N)$ essai $2-\boldsymbol{\Delta}:(E, N-n)-\mathbf{\square}:(E, n)$. $8 C:$ Kjeldahl-N content of dry matter $(\mathrm{g} N / 100 \mathrm{~g}$ D.M.) in lamina. 
tant de l'absorption pendant toute la repousse. Il est intéressant de souligner que cette disponibilité limitée au champ, au moins en fin de croissance, a été sans conséquence sur la production de biomasse aérienne : les auteurs indiquent que la croissance observée correspond à la croissance potentielle.

\section{CONCLUSION}

L'étude de la croissance, de l'assimilation du carbone et du métabolisme azoté à différents régimes de nutrition azotée est limitée par la difficulté de maintenir un niveau de carence comparable du début à la fin de l'expérimentation, même en conditions contrôlées. Ceci est cependant souhaitable étant donné la sensibilité de la répartition des assimilats carbonés à ce facteur (GASTAL \& Saugiter, 1986). Ce probleme technique est d'autant plus difficile à maîtriser que l'on provoque artificiellement des variations de vitesse de croissance en modulant l'intensité de l'éclairement ou la température. En ce sens, le protocole classique de culture à différentes concentrations en azote dans le milieu nutritif n'est pas satisfaisant.

Le travail présenté indique que la relation entre teneur en N-Kjeldahl et la biomasse de l'ensemble limbes et gaines n'est pas modifiée, en conditions de nutrition azotée non limitante, par la vitesse de croissance lorsque cette dernière dépend du niveau d'éclairement. Ceci est en accord avec les capacités rapides d'ajustement de l'absorption d'azote à la vitesse de croissance mesurées à l'échelle de la journée (GASTAL, 1984). Il serait nécessaire, pour une généralisation plus complète, d'étudier la validité de cette relation dans des conditions de température variées, étant donné la sensibilité des enzymes du métabolisme de l'azote à ce facteur de l'environnement. Ces résultats confirment cependant l'intérêt de cette relation comme critère d'appréciation de la disponibilité en azote du sol de prairie, ainsi que cela a été proposé par LEMAIRE \& SALETTE (1984). Les teneurs en nitrate, utilisées comme outil pour ce genre de diagnostic, peuvent certainement apporter des informations complémentaires par rapport à la teneur en $\mathrm{N}$ Kjeldahl. Mais, seules, elles n'offrent pas selon nous une sécurité suffisante: d'une part, leur disparition dans les plantes est très rapide et pratiquement totale pour un niveau de carence relativement modéré, d'où des teneurs au champ généralement très faibles, d'autre part, l'utilisation de fertilisants ammoniaconitriques ainsi que les dosages d'azote minéral dans le sol suggèrent qu'une partie de la nutrition azotée des graminées fourragères au champ pourrait passer par la forme ammoniacale.

En matière de qualité azotée des fourrages, la prise en considération de la fraction $\mathrm{N}$-organique soluble est nécessaire. Il apparaît que l'évolution de cette fraction en cas de carence en azote est très différente selon la partie de plante considérée. Là encore, des difficultés méthodologiques apparaissent : comment passer de l'azote organique soluble défini par la physiologie végétale à la fraction « utile» pour les ruminants ? Son efficacité chez l'animal dépend en partie du contenu en glucides de l'herbe. A ce niveau également, l'étude simultanée du métabolisme azoté et carboné de la plante se révèle déterminante.

Reçu le 13 mai 1985. Accepté le 4 décembre 1985.

\section{RÉFÉRENCES BIBLIOGRAPHIQUES}

Caloin M., Yu O., 1984. Analysis of the time course of change in nitrogen content in Dactylis glomerata L. using a model of plant growth. Ann. Bot., 54, 69-76.

Clement C. R., Hopper M. J., Jones L. H. P., 1978. The uptake of nitrate by Lolium perenne from flowing nutrient solution. 1. - Effect of $\mathrm{NO}_{3}^{-}$concentration. J. Exp. Bot., 29, 453-464.

Darwinkel A., 1975. Aspects of assimilation and accumulation of nitrate in some cultivated plants. Proefschrift, Landbouwhogeschool te Wageningen, $63 \mathrm{p}$.

Deinum B., 1984. Chemical composition and nutritive value of herbage in relation to climate. In : « The impact of climate on grass production and quality ». Proc. 10th gen. Meet. Eur. Grassl. Fed., 338-350.

Gastal F., 1984. Echanges de $\mathrm{CO}_{2}$ et croissance de la fétuque élevée en interaction avec la nutrition azotée. Thèse $3^{\mathrm{e}}$ Cycle, Orsay, $126 \mathrm{p}$.

Gastal F., Saugier B., 1986. Alimentation azotée et croissance de la fétuque élevée. I. - Assimilation du carbone et répartition entre organes. Agronomie, 6 (2).

Gounot M., 1982. Insertion de la morphogenèse dans les modèles de productivité primaire. Acta Oecol., Oecol. gener., 3 (1), 53-74.

Ingestad F., Lund A. B., 1979. Nitrogen stress in birch seedlings. 1. - Growth technique and growth. Physiol. Plant., 45, 137-148.

Lemaire G., Salette J., 1984. Relation entre dynamique de croissance et dynamique de prélèvement d'azote par un peuplement de graminées fourragères. I. - Etude de l'effet du milieu. Agronomie, $4(5), 423-430$.

Nye P. H., Tinker P. B., 1977. The mineral nutrition of single plant in soil, 187-242. In : "Solute movements in the soil-root system ". Studies in Ecology, 4, Blackwell Scientific Publications. 342 p.

Pate J. S., Atkins C. A., 1983. Xylem and phloem transport and the functional economy of carbon and nitrogen of a legume leaf. Plant Physiol., 71, 835-840.

Penning de Vries F. W. T., 1982. Crop production in relation to availability of nitrogen, 213-221. In : " Simulation of plant growth and crop production ». Wageningen, Pudoc I, 11, 308 p.

Robin P., Conejero G., Passama L., Salsac L., 1983. Evaluation de la fraction métabolisable du nitrate par la mesure in situ de sa réduction. Physiol. Vég., 21 (1), 115-122.

Robson M. J., Deacon M. J., 1978. Nitrogen deficiency in small closed communities of S24 ryegrass. II. - Changes in the weight and chemical composition of single leaves during their growth and death. Ann. Bot., 42, 1199-1213.

Salette J., Lemaire G., 1981. Sur la variation de la teneur en azote des graminées fourragères pendant leur croissance : formulation d'une loi de dilution. C. R. Acad. Sci., Paris, 292, 875-878.

Triboï-Blondel A. M., 1979. Dynamique comparée de l'absorption de l'eau et des nitrates par des plantules de blé. C. R. Acad. Sci., Paris, 288, série D, 1545-1548. 